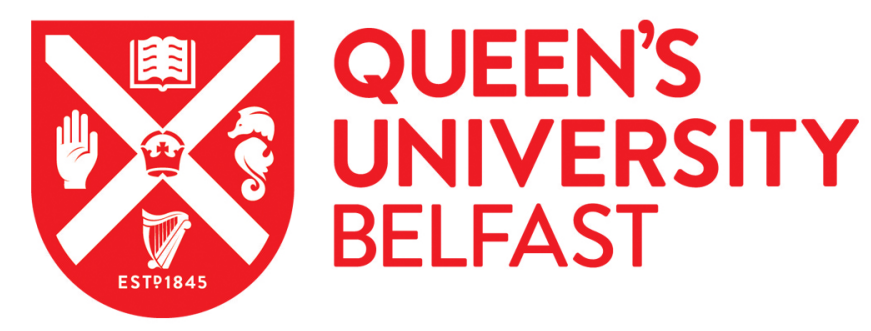

\title{
Comparative Profiling of Three Atheris Snake Venoms: A. squamigera, $A$. nitschei and $A$. chlorechis
}

Wang, H., Chen, X., König, E., Zhou, M., Wang, L., Chen, T., \& Shaw, C. (2018). Comparative Profiling of Three Atheris Snake Venoms: A. squamigera, A. nitschei and A. chlorechis. The Protein Journal, 37(4), $353-360$. https://doi.org/10.1007/s10930-018-9781-y

\section{Published in:}

The Protein Journal

\section{Document Version:}

Peer reviewed version

Queen's University Belfast - Research Portal:

Link to publication record in Queen's University Belfast Research Portal

\section{Publisher rights}

(C) Springer Science+Business Media, LLC, part of Springer Nature 2018. This work is made available online in accordance with the publisher's policies. Please refer to any applicable terms of use of the publisher.

\section{General rights}

Copyright for the publications made accessible via the Queen's University Belfast Research Portal is retained by the author(s) and / or other copyright owners and it is a condition of accessing these publications that users recognise and abide by the legal requirements associated with these rights.

Take down policy

The Research Portal is Queen's institutional repository that provides access to Queen's research output. Every effort has been made to ensure that content in the Research Portal does not infringe any person's rights, or applicable UK laws. If you discover content in the Research Portal that you believe breaches copyright or violates any law, please contact openaccess@qub.ac.uk. 


\section{Comparative profiling of three Atheris snake venoms: $A$. squamigera, $A$. nitschei and A. chlorechis}

He Wang ${ }^{\mathrm{a}, *}$, Xiaole Chen ${ }^{\mathrm{b}, *}$, Enrico König ${ }^{\mathrm{c}}$, Mei Zhou ${ }^{\mathrm{d}}$, Lei Wang ${ }^{\mathrm{d}}$, Tianbao Chen ${ }^{\mathrm{d}}$, Chris Shaw $^{\mathrm{d}}$

a. School of Integrative Medicine, Fujian University of Traditional Chinese Medicine, Fuzhou, Fujian, China

b. School of Pharmacy, Fujian Medical University, Fuzhou, Fujian, China

c. Laboratory of Synthetic and Structural Vaccinology, University of Trento, Trento, Trentino, Italy

d. Natural Drug Discovery Group, School of Pharmacy, Queen's University Belfast, Belfast, Northern Ireland, UK

* Corresponding author.

E-mail address: hwang11@qub.ac.uk (HeWang), leochen5139@fjmu.edu.cn (Xiaole Chen) 


\begin{abstract}
A proteomic and transcriptomic comparative analysis of the venoms of three Atheris species (A. squamigera, A. nitschei and A. chlorechis) was carried out by size exclusion liquid chromatography, gel electrophoresis, mass spectrometry, and mRNA sequencing. The improved proteomic profiling utilised in this work was combined with transcript studies, advancing our insights into venom composition, protein distribution and inter-species variation among the three bush vipers. Crude venoms of all three samples contained at least 10-20 protein components, ranging in size from $<=3 \mathrm{KDa}$ to $>98 \mathrm{KDa}$. Both approaches yielded converging overall information, pointing to phospholipases, disintegrins, serine proteases and metalloproteases as the major toxin classes, which are likely to explain the local and systemic symptoms observed in envenomation by Atheris genus. Being considered as the main factors involved in the distinct venom-induced pathologies, these identified snake venom proteins are of particular interest in terms of understanding their physiological and biological function as well as for their contribution in potential medical treatments.
\end{abstract}

\title{
Key words:
}

Atheris, proteomics, snake venom, toxins 


\section{List of Abbreviation}

A. chlorechis

A. nitschei

A. squamigera

BCA

BLAST

CDD

cDNA

CHCA

MALDI-TOF

mRNA

MS

NCBI

NUP

PBS

PCR

pHpG

RACE

SDS-PAGE

SEC

SMART

SVMP

SVSP
Atheris chlorechis

Atheris Nitschei

Atheris squamigera

Bicinchoninic acid

Basic Local Alignment Search Tool

Conserved domain database

Complementary DNA

Alpha-cyano-5-hydroxycinnamic acid

Matrix-assisted laser desorption ionization, time-of-flight

Messenger RNA

Mass spectrometry

National Centre of Biotechnology Information

Nested universal primer

Phosphate buffered saline

Polymerase chain reaction

Poly-histidine and poly-glycine

Rapid Amplifiction of cDNA Ends

Sodium dodecyl sulfate polyacrylamide gel electrophoresis

Size exclusion chromatography

Switching Mechanism At 5' end of RNA Transcript

Snake venom metalloproteinases

Snake venom serine proteinases 


\section{Introduction}

Viper snakebites account for the majority of deadly envenomation accidents all over the world, caused by the action of venom toxins [1]. Viper venoms are usually composed of toxins belonging to several protein families: snake venom serine proteinases (SVSP), phospholipases, snake venom metalloproteinases (SVMP), L-amino acid oxidases as well as non-enzymatic proteins such as disintegrins and protease inhibitors [2-3]. For species-specific snake venoms, their intraspecific variability in protein composition, distinct distribution of proteomic components and the presence of isoforms are common problems that one has to face when the development of a specific antidote is needed [4]. However, this issue has encouraged the isolation and characterisation of snake venom components, in particular proteins, at a large scale to improve the quality of therapeutic strategies. Moreover, snake venom proteins are known to be of considerable physiological, biological and medical value, making them an important resource of bioactive molecules with great potential for new drug development [5].

The genus Atheris (bush vipers) belongs to the family Viperidae and occurs in the African rainforests of the tropical subsaharan regions [6]. A brief description of the three species studied here is listed in Table 1[6]. These snakes have been held responsible for serious bites in quite a few of cases [7-10]. Envenomation upon bites of Atheris are clinicallycharacterised by coagulation disturbance (prolonged coagulation time and non-clotting of blood), bleeding and haemorrhaging, local pain, and oedema $[8,11]$. Some victims developed acute renal failure ${ }^{[9]}$ and even hypertension [10]. Symptomatic replacement therapy is commonly applied due to the absence of an Atheris specific antivenom [7-8,10]. However, treatment of A. chlorechis envenomation using a non-specific antiserum (FAV AFRIQUE) described by Top [9], appeared to be efficient in neutralising the toxins, although knowledge about the antidotal effect was incomplete at that time. Interestingly, an antivenom against 
another viper genus, Echis, has been reported to be effective in a case of human envenomation by A. squamigera [12]. The venom compositions of species from the genus Atheris have not been fully elucidated yet. Biochemical and biological investigations of venom from $A$. squamigera revealed the existence of some components causing haemocoagulation disorders [8]. These studies emphasised the need for further investigation on Atheris venom toxins, in particular the identification of venom components, the elucidation of mechanisms leading to venom toxicity as well as the quantitative and qualitative characterization in terms of the venom protein pattern.

The lack of information may derive from some difficulties and the limits of analytical techniques, which require alternative approaches to separate complex protein mixture. However, recent developments in proteomics provide helpful tools to overcome this issue and could thus help to improve a comprehensive analysis of crude snake venom and identification of the containing proteins from such complex mixture [13-14]. Therefore, proteomic studies of snake venoms could give new insight into the global venom proteome, focusing on the protein expression pattern and functional characterisation. To explain the molecular basis of envenomation pathophysiologies, our comparative, proteomic analyses allowed us to hypothesise about the roles of different proteins in toxicological processes, thereby providing a way to understand their clinical significance. In this work, the characterisation and comparative study of the venom proteomes of three Atheris bush vipers by use of SDSPAGE, chromatographic fractionation and mass spectrometry, is described. In addition, the venom gland complementary DNA libraries were constructed and molecular cloning of the main venom transcripts was performed. We previously reported the cloning and characterisation of three novel cDNAs encoding disintegrin precursors [15] and three novel cDNAs encoding phospholipase A2 precursors [16] from the venoms of the three Atheris vipers from the present study using the same molecular cloning strategy. The existing set of 
venom transcripts derived from the three Atheris species were used and combined with the proteomic data from the present study aiming to elucidate a general, diagnostic profile of different Atheris venoms.

\section{Materials and methods}

\section{Preparation of venoms}

$100 \mathrm{mg}$ of pooled venom samples from three bush viper species (Atheris squamigera, Atheris nitschei and Atheris chlorechis) were purchased from Latoxan, Rosans, France. All venoms were stored lyophilised at $-20^{\circ} \mathrm{C}$ until used.

\section{Size-exclusion liquid chromatography and identification}

To obtain a general elution profile, venoms were analysed via size exclusion chromatography (SEC) using an ÄKTAprime ${ }^{\mathrm{TM}}$ plus system (GE Healthcare) and a Superdex 75 10/300 GL column. Each crude venom (10-12mg) was resuspended in $1 \mathrm{ml}$ of buffer $\left(0.05 \% / 30 \% / 70 \%(\mathrm{v} / \mathrm{v} / \mathrm{v}) \mathrm{TFA} /\right.$ acetonitrile $\left./ \mathrm{H}_{2} \mathrm{O}\right)$ and $400 \mu \mathrm{l}$ were injected into the column equilibrated with the same buffer. The separation was carried out under isocratic conditions at a flow rate of $0.5 \mathrm{ml} / \mathrm{min}$ over $60 \mathrm{~min}$ and absorbance was detected at $280 \mathrm{~nm}$.

Identification of proteins or peptides present in the collected fractions was performed using a Voyager DE Matrix-assisted laser desorption/ionization-Time of Flight (MALDITOF) mass spectrometer under the control of the Applied Biosystems Voyager System TM (Applied Biosystems, Framingham, MA). One microliter of each fraction was applied on a 100 well sample-loading plate (Applied Biosystems, Framingham, MA) and pre-coated with alpha-cyano-5-hydroxycinnamic acid (CHCA, $10 \mathrm{mg} / \mathrm{ml}$ in $70 \%$ acetonitrile $/ 0.01 \%$ trifluoroacetic acid). The spectrometer operated in positive reflector and in linear modes, spectra were acquired in the mass range of 500 to 20,000 Da. 


\section{SDS-PAGE and protein identification}

Venom samples were diluted to a final concentration of $2.0 \mathrm{mg} / \mathrm{ml}$ crude venom in phosphate buffered saline (PBS). For quantification of total protein present in the crude venom samples the Bicinchoninic acid protein assay (BCA Protein Assay kit, Thermo Scientific Pierce, UK) was used. NuPage 4-12\% pre-cast acrylamide gels (Invitrogen, UK) were loaded with $32 \mu \mathrm{g}$ of each venom sample, run in 1x SDS (sodium dodecyl sulfate) running buffer for $35 \mathrm{~min}$ at $200 \mathrm{~V}$, and proteins were stained with Coomassie Brilliant Blue (Invitrogen, UK). The relative amounts of stained protein bands were measured by densitometry using the Gel-Pro Analyzer 4.0 software (Media cybernetics, MA, USA). In addition, all SEC fractions were assessed for their protein concentration using the BCA Protein Assay kit (Thermo Scientific Pierce, UK) and subsequently loaded on a NuPage 4$12 \%$ pre-cast acrylamide gel for SDS-PAGE.

Protein bands of interest were excised according to their density and distribution and digested overnight using trypsin (Sigma, UK). The resultant tryptic peptides were subjected to an LCQ Fleet Ion Trap MS (Thermo Fisher, UK) with a Phenomonex Jupiter C18 column $(5 \mu \mathrm{m}, 150 \times 2 \mathrm{~mm})$ using a gradient of $0-60 \%$ acetonitrile/ $0.1 \mathrm{M}$ acetic acid at a flow rate of $0.2 \mu \mathrm{l} / \mathrm{min}$. Eluted peptides were analysed in a data-dependent mode to select multicharged peptides for further MS/MS fragmentation of the ten most abundant ions.

\section{Construction of venom-derived cDNA libraries}

Complementary DNA libraries were constructed by reverse transcription of polyadenylated mRNA extracted from $5 \mathrm{mg}$ of lyophilized venom using Dynabeads ${ }^{\circledR}$ M DIRECT ${ }^{\mathrm{TM}}$ Kit (Dynal Biotech, UK). A SMART ${ }^{\mathrm{TM}}$ Rapid Amplification of cDNA ends (RACE) cDNA Amplification kit (BD Clontech, UK), both 5'-and 3' ends cDNA ends were synthesized using SMART (Switching Mechanism At 5' end of RNA Transcription) cDNA synthesis technology. For the reactions, a nested universal primer (NUP) and degenerated sense 
primers were applied. Two types of primers were applied: (1) homologous primers designed on the basis of conserved 3'-untranslated region of analogue snake toxins identified from evolutionary related species of the same subfamily Viperinae like Bitis and Echis ${ }^{[15-16]}$; (2) degenerated primers for reverse transcription based on the identified protein sequences obtained by MS/MS fragmentation. The obtained cDNA fragments with the expected size were purified and used for transformation of E. coli cells using the pGEM-T vector system for subsequent blue-white screening according to manufacturer's manual (Promega, UK). After transformation, a PCR of the plasmid DNA obtained from positive E. coli cells was performed, and the PCR fragments were purified with a DNA Extraction kit (RBD Bioscience) for subsequent sequencing using a 3730 DNA sequencer (Applied Biosystems).

\section{Bioinformatic analysis}

The BLAST tool of the National Centre for Biotechnology Information (NCBI) was employed for protein identification of all obtained sequences and to exclude possible contaminations. Multiple sequence alignments of the DNA and amino acid sequence were performed using the Vector NTI software.

\section{Result}

\section{The venom compounds of three Atheris species}

Each of the three Atheris venom samples exhibited distinct chromatographic profiles using SEC (Figure 1). The observed $\mathrm{m} / \mathrm{z}$ ratios from MALDI-TOF analysis of the collected SEC fractions are shown in Table 2.

The most abundant components eluted at a retention time between 20 and $35 \mathrm{~min}$ corresponding to protein sizes of 43-0.2 $\mathrm{kDa}$. The collected fractions contained polypeptides of about 7-14 kDa. Finally, short peptides ranging from 2-4.5 kDa, although not visable after SDS-PAGE, were detectable through MALDI-TOF MS analyses. 
SDS-PAGE was run to compare the protein pattern of the three crude venoms. Comparable to SEC, different patterns in terms of band intensity and distribution were observed (Figure 2). A number of protein bands occurred in all three samples, ranging in size from approximately 3-65 $\mathrm{kDa}$, but also a weak band at a size of ca. $100 \mathrm{kDa}$ was present in the samples of $A$. squamigera and A. nitschei. The selected protein bands were cut and prepared for in-gel digestion. Based on the identified peptide sequences obtained from subsequent tandem mass spectrometry analyses, the presences of the three protein families disintegrin, phospholipase $\mathrm{A}_{2}(7-14 \mathrm{kDa}$ ) and SVMP (ca. 40-55 kDa) in all of the three Atheris venoms samples confirmed in this study(Table 3). Moreover, identified peptides suggesting the presence of SVSP in the venom of A. squamigera and A. nitschei, whereas this protein family was absent in A. chorechis.

Some low molecular weight peptides $(<3,000 \mathrm{Da})$ were found in both $A$. squamigera and A. chlorechis venoms, but not in the venom of A. nitschei. However, a cluster of small peptides was detected from all venoms (Table 2). The primary structures identified by MS/MS sequencing did not show any sequence homology with previously published viper peptide toxins, such as myotoxins and neurotoxins.

A qualitative and quantitative distribution of the different venom protein families identified in the three Atheris venoms is shown in Table 3, pointing to PLA2, disintegrins, serine proteases and metalloproteases as the major toxin compounds.

\section{General description of venom transcripts}

The species-specific cDNA libraries have been constructed from the venoms of $A$. squamigera, A. nitschei and A. chlorechis with a molecular cloning strategy developed in our laboratory. To gather the maximum amount of information about the putative secreted proteins, selected sequences presented in Table 3 were used to design degenerated primers for the cloning of the venom-derived cDNA libraries. These fragments played an extensive role 
in the genomic investigation for a homologue primer design as well. As a result, three novel Group II PLA2 precursor transcripts were consistently cloned and named PLA2-A.C., PLA2A.N. and PLA2-A.S, respectively (Genbank accession numbers KP119682 to KP119684).

In this research, the signal peptide and part of the propeptide sequence of SVMP has been cloned from the venom-derived cDNA library of Atheris squamigera (Figure 3). According to the CDD (conserved domain database) analysis, the propeptide can be classified as a member of the Pep_M12B_propeptide group SVMP [17]. Although the whole sequence of SVMP was not cloned successfully with our standard approach, a platelet aggregation inhibiting activity of the corresponding snake venom fraction could approve the presence of this protein family.

\section{Discussion}

The three species of Atheris (A. squamigera, A. nitschei and A. chlorechis) were studied using SDS-PAGE of crude venoms, chromatographic profiling (SEC) and an established molecular cloning strategy. Combining all methodologic approaches, at least 10-20 protein components, ranging in size from $\leqslant 3 \mathrm{kDa}$ to $>98 \mathrm{kDa}$, were apparent in the crude venoms. Several components are common to all venoms although with quantitative differences, such as disintegrins, phospholipases and SVMPs, whereas some identified components were species-specific. These differences among species could be the result of diverse factors such as geographic region, ecological factors, prey patterns, evolutionary traits, ontogenetic or individual variability, sexual recombination and other genetic factors as well as age and the storage conditions of a venom sample [3, 24].

A summary of venom gland transcripts (cDNA) found in the Atheris genus, including those previously reported and the present study is presented in Table 4. The nucleotide and deduced amino acid sequences of all three PLA2 have been reported previously [16]. We have also reported the first genomic sequences of three cDNA encoding disintegrin 
precursors form Atheris venoms [15], named DS-A.C., DS-A.N. and DS-A.S. (EMBL Nucleotide Sequence Database: accession numbers HF543862-HF543864). At the molecular level, the proteomic and transcriptomic datasets exhibit high compositional concordance. These data emphasize the value of combining two approaches to acquire a more complete understanding of the toxicological profile of the snake venom.

It is noteworthy that the venom of $A$. squamigera contains a peptide with a unique structure in having high repetition of the single amino acids, histidine and glycine ( $\mathrm{pHpG}$ ) probably leads to specific biological and pathological effects [18]. Part of the $\mathrm{pHpG}$ show significant sequence identity with the short enzyme inhibitor from Echis ocellatus venom that specifically targets the zinc-metalloproteinase [19]. Chemically synthesised replicates of pHpG peptides will be carried out for a spectrum of bioactive assays in the future work.

As an example of their vast potential and as an invaluable source of biological compounds, the venoms of vipers are of great significance. In snakebites, the tissues or vessels of prey are pierced by sharp fangs and an often massive volume of venom will be injected [20]. With the assistance of enzymes/toxins in their venoms, snakes can easily kill prey by chemical poisoning as well as by the normal mechanical methods [21]. The venom components usually have high proteolytic activities for pre-digestion of prey that can also provide assistance to the normal mechanical method. Thus the enzymatic activities of venoms, especially those of some proteinases, are closely correlated with prey patterns [22-23]. The Atheris genus contains species that, in common with other species of Viperidae snakes, usually feed upon amphibians, lizards and even larger prey-birds and snakes (Table 1) [6], suggesting the importance of the enhanced pre-digestive activities of their venoms, although Atheris species are not well-characterized for their venom toxins. However, evidence from the present study confirms that Atheris venoms contain powerful components, such as SVSP, phospholipases, 
SVMP and the tissue-destroying disintegrins, making the snakes potentially lethal for humans while promoting initial digestion of the natural preys of bush vipers.

Moreover, the clinical symptoms observed following envenomation by Atheris vipers, could obviously occur due to several families of proteins identified in this work and previous studies from our lab [15-16]. Local haemorrhage, pain and oedema are mainly caused by enzymatic components, and severe coagulopathy could result from the actions of disintegrins that mainly contribute to the platelet aggregative inhibition. Although there is little information on the clinical manifestations of Atheris viper bites [12], its critical features would be closely correlated with the protein composition. It was therefore important to perform further studies on the structural, biochemical and pharmacological characteristics of the venom proteins in order to give a better insight into their functional relationships and their roles in discrete envenomation.

The comparative biochemical studies on three venoms studied in the current work (A. squamigera, A. nitschei and A. chlorechis) indicate that intriguing variations exist in composition and concentration of venom protein molecules that play distinct roles. The identification of variable venom compositions may contribute to overcome the obstacles for the development of effective clinical therapies, because the antidote available for one species could be either partially effective or ineffective against the toxicity of another species [2526]. In the case of Atheris envenomation, antivenom administration is even more complicated because specific antidotes are lacking and thus only general substances or antivenoms for other species can be applied. Consistently, optimisation of therapeutic envenomation treatment, a more comprehensive knowledge about the snake venom heterogeneity and detailed individual, species-specific molecular characterisations are of needed. 


\section{Acknowledgement}

The project was kindly supported by the Natural Science Foundation of Fujian Province, China (Grant No. 2015J05162), the Science Foundation of Education Department of Fujian Province, China (Grant No. JAT160247), the Young talent project of Health Department of Fujian Province, China (Grant No. 2017-1-69), the National Natural Science Foundation, China (Grant No. 81402842) and the Joint Funds for the innovation of science and Technology, Fujian province (Grant No.2016Y9048).

\section{Conflict of Interest statement}

The authors declare that they have no conflict of interest.

\section{Ethical statement}

All applicable international, national, and/or institutional guidelines for the care and use of animals were followed. 


\section{References}

[1] Pandey, D. P., Vohra, R., Stalcup, P., \& Shrestha, B. R. (2016). A season of snakebite envenomation: presentation patterns, timing of care, anti-venom use, and case fatality rates from a hospital of southcentral nepal. Journal of Venom Research, 7, 1-9.

[2] Alapegirón, A., Sanz, L., Escolano, J., Floresdíaz, M., Madrigal, M., \& Sasa, M., et al. (2008). Snake venomics of the lancehead pitviper bothrops asper: geographic, individual, and ontogenetic variations. Journal of Proteome Research, 7(8), 3556.

[3] Birrell, G. W., Earl, S. T., Wallis, T. P., Masci, P. P., De, J. J., \& Gorman, J. J., et al. (2007). The diversity of bioactive proteins in australian snake venoms. Molecular \& Cellular Proteomics Mcp, 6(6), 973.

[4] Tanaka, G. D., Furtado, M. D. F. D., Portaro, F. C. V., Sant'Anna, O. A., \& Tambourgi, D. V. (2010). Diversity of micrurus snake species related to their venom toxic effects and the prospective of antivenom neutralization. Plos Neglected Tropical Diseases, 4(3), e622.

[5] Samy R P, Sethi G, Lim L H(2016) A brief update on potential molecular mechanisms underlying antimicrobial and wound-healing potency of snake venom molecules. Biochemical Pharmacology 115:1-9.

[6] Spawls S, Branch W R(1995) The dangerous snakes of Africa: natural history, species directory, venoms and snakebite

[7] Knoepffler L P(1965) Autoobservation d'envenimation par morsure d'Atheris sp. Toxicon 2(4):275-276.

[8] Mebs, D., Holada, K., Kornalík, F., Simák, J., Vanková, H., \& Müller, D., et al. (1998). Severe coagulopathy after a bite of a green bush viper (atheris squamiger): case report and biochemical analysis of the venom. Toxicon Official Journal of the International Society on Toxinology, 36(10), 1333. 
[9] Top, L. J., Tulleken, J. E., Ligtenberg, J. J., Meertens, J. H., Ts, V. D. W., \& Zijlstra, J. G. (2006). Serious envenomation after a snakebite by a western bush viper (atheris chlorechis) in the netherlands: a case report. Netherlands Journal of Medicine, 64(5), 153-156.

[10] Valenta, J., Stach, Z., Fricova, D., Zak, J., \& Balik, M. (2008). Envenoming by the viperid snake proatheris superciliaris : a case report. Toxicon Official Journal of the International Society on Toxinology, 52(2), 392-394.

[11] Favreau, P., Cheneval, O., Menin, L., Michalet, S., Gaertner, H., \& Principaud, F., et al. (2010). The venom of the snake genus atheris contains a new class of peptides with clusters of histidine and glycine residues. Rapid Communications in Mass Spectrometry, 21(3), 406412.

[12] Mallow, D., Ludwig, D., Nilson, G., Mallow, D., Ludwig, D., \& Nilson, G. (2003). True vipers: natural history and toxinology of old world vipers. True Vipers Natural History \& Toxinology of Old World Vipers.

[13] Li, S., Wang, J. X., Ren, Y., Wang, N., Zhao, K., \& Chen, X., et al. (2004). Proteomic characterization of two snake venoms: naja naja atra and agkistrodon halys. Biochemical Journal, 384(1), 119-127.

[14] Calvete, J. J., Petras, D., Calderóncelis, F., Lomonte, B., Encinar, J. R., \& Sanzmedel, A. (2017). Protein-species quantitative venomics: looking through a crystal ball. Journal of Venomous Animals \& Toxins Including Tropical Diseases, 23(1), 27.

[15] Wang, H., Chen, X., Wang, L., Chen, W., Zhou, M., \& Chen, T., et al. (2013). Cloning and characterisation of three novel disintegrin precursors from the venoms of three atheris species: atheris chlorechis, atheris nitschei and atheris squamigera. Toxicon, 71, 31-40.

[16] Wang, H., Chen, X., Zhou, M., Wang, L., Chen, T., \& Shaw, C. (2016). Molecular characterization of three novel phospholipase a2proteins from the venom ofatheris chlorechis, atheris nitscheiandatheris squamigera. Toxins, 8(6), 168. 
[17] Rawlings N D, Barrett A J.(1995) Evolutionary families of metallopeptidases Methods in Enzymology 248(248):183.

[18] Wang, H., Wang, L., Zhou, M., Chen, T., \& Shaw, C. (2010). Molecular cloning and deduced organization of the phpg/cnp precursor from the venom of the african forest viper, atheris squamigera. Regulatory Peptides, 164(1), 35-35.

[19] Wagstaff, S. C., Favreau, P., Cheneval, O., Laing, G. D., Wilkinson, M. C., \& Miller, R. L., et al. (2008). Molecular characterisation of endogenous snake venom metalloproteinase inhibitors. Biochemical \& Biophysical Research Communications, 365(4), 650-656.

[20]Cundall D.(2009) Viper fangs: functional limitations of extreme teeth Physiological \& Biochemical Zoology Pbz 82(1):63.

[21] Mackessy S P.(2010) Evolutionary trends in venom composition in the Western Rattlesnakes ( Crotalus viridis, sensu lato): Toxicity vs. tenderizers. Toxicon 55(8):1463-74. [22] Barlow, A., Pook, C. E., Harrison, R. A., \& Wüster, W. (2009). Coevolution of diet and prey-specific venom activity supports the role of selection in snake venom evolution. Proceedings Biological Sciences, 276(1666), 2443-2449.

[23] Moura-Da-Silva, A. M., Almeida, M. T., Portes-Junior, J. A., Nicolau, C. A., Francisco, G. N., \& Valente, R. H. (2016). Processing of snake venom metalloproteinases: generation of toxin diversity and enzyme inactivation:. Toxins, 8(6), 183.

[24] Cipriani, V., Debono, J., Goldenberg, J., Tnw, J., Arbuckle, K., \& Dobson, J., et al. (2017). Correlation between ontogenetic dietary shifts and venom variation in australian brown snakes (pseudonaja). Comparative Biochemistry \& Physiology Part C Toxicology \& Pharmacology, 197, 53-60.

[25] Calvete, J. J., Sanz, L., Angulo, Y., Lomonte, B., \& Gutiérrez, J. M. (2009). Venoms, venomics, antivenomics. Febs Letters, 583(11), 1736-1743. 
[26] Gutiérrez, J. M., Solano, G., Pla, D., Herrera, M., Segura, Á, \& Vargas, M., et al. (2017). Preclinical evaluation of the efficacy of antivenoms for snakebite envenoming: state-of-theart and challenges ahead:. Toxins, 9(5), 163. 


\section{Figure Legends}

Figure 1. Venom profile of three Atheris species(A) Atheris squamigera, (B) Atheris nitschei, (C) Atheris chlorechis. The electrophoresis analysis of fractions collected from the sizeexclusion liquid chromatography is displayed on top of each spectrum. Molecular masses of chromatographic fractions are listed in Table 2.

Figure 2. The electrophoretic patterns of venom proteinsobtained using polyacrylamide gels (4-12\%). A.C.-Atheris chlorechis, A.N.-Atheris nitschei, A.S.-Atheris squamigera, MMMolecular Weight Markers with sizes were indicated on the right. Identified proteins bands were numbered from 1 to 16 . Molecular masses, sequence tags and database hits are listed in Table 3.

Figure3. (A) Nucleotide sequence and the deduced amino acid sequence of the open-reading frame (ORF) of the clones encoding partial of the metalloproteinase precursor (Metallopretease-1). The putative signal sequence is double underlined. Only part of the sequence identified belonging to the Pep_M12B_propeptide family has been cloned. (B) Amino acid sequence multiple alignments of Metalloprotease-1 precursor found in this work and other M12B metalloproteases from the Genbank database. The accession number of each protein is marked at the front of array. Identical bases are shaded in black and consensus bases are shaded gray. The amino acid is numbering at the top. 


\section{Table Legends}

Table 1. Description of the three Atheris snake species.

Table 2. Corresponding identification of chromatoghraphic eluted portions by Matrixassisted laser desorption/ionization-Time of Flight (MALDI-TOF) mass spectrometry: (a) Atheris squamigera, (b) Atheris nitschei, (c) Atheris chlorechis.

Table 3. Summary of the relative quantities of Atheris snake venom proteins identified by structural class through the combined data derived from electrophoresis, in-gel digestion and mass spectrometric strategies. Average abundance was calculated according to the electrophoretic pattern obtained for each snake venom as shown in Figure 3.3.4.

Table 4. A catalog of three Atheris venom gland cDNA for mainly secretary proteins 
Table 1

\begin{tabular}{|c|c|c|c|c|}
\hline Species & Common name * & Subspecies & $\begin{array}{l}\text { General prey } \\
\text { base }\end{array}$ & Geographic range \\
\hline $\begin{array}{l}\text { A.squamige } \\
\text { ra }\end{array}$ & Variable bush viper & 0 & $\begin{array}{l}\text { Rodents, lizards } \\
\text { and amphibians } \\
\text { and snakes }\end{array}$ & $\begin{array}{l}\text { Central Africa, from } \\
\text { Nigeria to Uganda and } \\
\text { Angola }\end{array}$ \\
\hline A.nitschei & Great Lakes bush viper & $\begin{array}{l}\text { 1(A.n.nitschei } \\
\text {,A.n.rungwee } \\
\text { nsis) }\end{array}$ & $\begin{array}{l}\text { Rodents, lizard } \\
\text { (especiallychameleo } \\
\text { ns) and amphibians }\end{array}$ & $\begin{array}{l}\text { From East Africa, Uganda to } \\
\text { the Central Lake Tanganyika }\end{array}$ \\
\hline $\begin{array}{l}\text { A.chlorechi } \\
\text { s }\end{array}$ & Western bush viper & 0 & $\begin{array}{l}\text { Rodents, lizards } \\
\text { and amphibians } \\
\text { (tree frogs) }\end{array}$ & $\begin{array}{l}\text { Western Africa, including } \\
\text { Guinea-Bissau, Guinea, } \\
\text { Sierra Leone, et al. }\end{array}$ \\
\hline
\end{tabular}

*Only the most frequently-used common names are shown for each species. 


\section{Table2}

(A)

\begin{tabular}{ll}
\hline Fraction Time (min) & M/Z ratio of components \\
\hline $17-18$ & $6805 / 7141 / 13840$ \\
$19-20$ & $4945 / 7142 / 13840$ \\
$21-24$ & $4943 / 7119$ \\
$29-30$ & $1355 / 1703 / 2842 / 3170 / 3597$ \\
$31-32$ & $1355 / 1703 / 2344 / 2788 / 2844$ \\
$33-34$ & $1345 / 2100 / 2277 / 2758 / 2798$ \\
$35-36$ & $1523 / 2136 / 2369$ \\
$37-40$ & $1480 / 2135$ \\
\hline
\end{tabular}

(B)

\begin{tabular}{ll}
\hline Fraction Time (min) & $\mathbf{M} / \mathbf{Z}$ ratio of components \\
\hline $21-22$ & $7071 / 13975$ \\
$23-24$ & $4721 / 7084 / 13965$ \\
$25-26$ & $4727 / 7071$ \\
$29-30$ & $3311 / 4727$ \\
$35-36$ & $2787 / 3312 / 4718$ \\
$37-38$ & $1263 / 2609 / 2788 / 3311$ \\
$47-48$ & $1028 / 1263 / 2607$ \\
$49-50$ & $1027 / 1262$ \\
\hline
\end{tabular}

(C)

\begin{tabular}{ll}
\hline Fraction Time (min) & M/Z ratio of components \\
\hline $17-18$ & 13961 \\
$19-20$ & $6985 / 7012 / 13964$ \\
$21-22$ & $6989 / 7011 / 13961$ \\
$35-36$ & $3515 / 6985$ \\
$37-38$ & $2357 / 3519$ \\
$39-40$ & $2357 / 2961 / 3518$ \\
$47-48$ & $1061 / 2435$ \\
$49-50$ & 2456 \\
\hline
\end{tabular}


Table 3

\begin{tabular}{|c|c|c|c|c|}
\hline $\begin{array}{l}\text { Band } \\
\text { NO. }\end{array}$ & $\begin{array}{l}\text { Molecular } \\
\text { Mass } \\
\text { (KDa) }\end{array}$ & MS/MS Sequencing & Identification & $\begin{array}{l}\text { Snake } \\
\text { species }\end{array}$ \\
\hline 1 & $<3-5$ & --- & Short peptides & A.squamigera \\
\hline 2 & $\sim 6-7$ & FINSGTICK & Disintegrin & \\
\hline 3 & $\sim 14$ & NLFQFR & PLA2 & \\
\hline 4 & $\sim 20$ & --- & Unknown & \\
\hline 5 & $\sim 25-45$ & LFDYSVCR & SVSP & \\
\hline 6 & $\sim 47-60$ & LPCAPEDIK & SVMP & \\
\hline 7 & $\sim 6-7$ & NSPHPCCDPVTCK & Disintegrin & A.nitschi \\
\hline 8 & $\sim 12-14$ & NPYK & $\begin{array}{l}\text { Dimeric } \\
\text { Disintegrin }\end{array}$ & \\
\hline 9 & $\sim 14$ & NLFQFGSMIK & PLA2 & \\
\hline 10 & $\sim 25-45$ & IFDYSICR & SVSP & \\
\hline 11 & $\sim 45-55$ & VNGEPVVLHLEK & SVMP & \\
\hline 12 & $<3-5$ & --- & Short peptides & A.chorechis \\
\hline 13 & $\sim 6-7$ & FMNSGTICK & Disintegrin & \\
\hline 14 & $\sim 12-14$ & --- & $\begin{array}{l}\text { Unkown(Big } \\
\text { possible be } \\
\text { dimeric } \\
\text { Disintegrin) }\end{array}$ & \\
\hline 15 & $\sim 14$ & PQDDTNR & PLA2 & \\
\hline 16 & $\sim 30-45$ & LHPWVEGESGECCDK & SVMP & \\
\hline
\end{tabular}


Table 4

\begin{tabular}{|c|c|c|c|c|c|c|c|c|}
\hline Snake species & Sequence name & Protein family & Description & Genbank $^{1}$ & $\mathbf{M W}^{2}$ & Novel cDNA ${ }^{3}$ & Function & Reference \\
\hline \multirow[t]{4}{*}{ A.squamigera } & pHpG peptides & Poly $G$ peptides & Full-length & Unpublished & 2338 & $\mathrm{Y}$ & Induced haemorrhage & $\begin{array}{l}\text { Favreau et al, 2007; } \\
\text { Wagstff et al, } 2008\end{array}$ \\
\hline & DS-A.S. & Disintegrin & Full-length & HF543864 & 7042 & $\mathrm{Y}$ & Platelet inhibitor & Wang et al, 2013 \\
\hline & PLA2-A.S. & Phospholipase $\mathrm{A}_{2}$ & Full-length & KP119684 & 13840 & Y & Edema inducer & Wang et al, 2016 \\
\hline & Metallopretease-1 & Metallopretease & Partial & Unpublished & $\mathrm{NA}^{4}$ & $\mathrm{Y}$ & Haemorrhagic & This Work (Figure 4.) \\
\hline \multirow[t]{2}{*}{ A.nitschi } & DS-A.N. & Disintegrin & Full-length & HF543863 & 7071 & $\mathrm{Y}$ & Platelet inhibitor & Wang et al, 2013 \\
\hline & PLA2-A.N. & Phospholipase $\mathrm{A}_{2}$ & Full-length & KP119683 & 13975 & $\mathrm{Y}$ & Edema inducer & Wang et al, 2016 \\
\hline \multirow[t]{2}{*}{ A.chorechis } & DS-A.C. & Disintegrin & Full-length & HF543862 & 7012 & $\mathrm{Y}$ & Platelet inhibitor & Wang et al, 2013 \\
\hline & PLA2-A.C. & Phospholipase $\mathrm{A}_{2}$ & Full-length & KP119682 & 13964 & Y & Edema inducer & Wang et al, 2016 \\
\hline
\end{tabular}

1Genbank, NR database accession number

$2 \mathrm{MW}$, molecular mass of the mature protein

3Novel cDNA, Y=yes

${ }^{4} \mathrm{NA}$, Not Available 